\title{
Research on fire source localization in confined space based on the fire characteristic physical quantity information
}

\author{
Fan Yang ${ }^{1}$, Zhuoyuan $\mathrm{Cai}^{2}{ }^{2}$ (D), Lei $\mathrm{Su}^{1}$, Yongduan $\mathrm{Xue}^{2}$, Yu Shen ${ }^{1}$, and Junjie Wang ${ }^{2}$ \\ ${ }^{1}$ Hubei Electric Power Research Institute, Wuhan 430077, PR China \\ 2 Department of New Energy, China University of Petroleum (East China), Qingdao 266580, PR China
}

Received: 24 October 2022 / Accepted: 3 January 2022

\begin{abstract}
Fire source localization is of great significance to the firefighting and evacuation. In order to resolve the problem of precise localization of fire source in confined space, a method based on the diffusion law of hot smoke flow in the early stage of fire is proposed in this paper. According to the fire characteristic physical quantity information collected by the sensor array, the relative variability correlation degree is used to obtain the signal time delays between the sensor units. Then, the direction angle of the sensor units and fire source can be determined through the geometric relationship, and the angular localization principle is used to obtain the fire source localization results. Finally, according to the fire source localization results obtained in different sets of time delays, the localization estimation area is selected based on the dynamic clustering, and the center of this area is output as the comprehensive localization result. The testing results show that this method performs well and achieves a high localization accuracy.
\end{abstract}

Keywords: Fire source localization / confined space / fire characteristic physical quantity / dynamic clustering / localization accuracy

\section{Introduction}

Building fire is an abnormal event that causes social, economic, and ecological damage [1]. Fire detection is an essential requirement for emergency response to save life and property. At present, fire extinguisher system mostly uses fire sprinklers to protect the monitoring area [2]. This method can provide an effective protection for the monitoring area, but it cannot focus on the location of the fire source. Due to the waste of water and low extinguishing efficiency, it is easy to cause unnecessary property losses [3]. In addition, for some multi-compartment buildings, such as commercial and office buildings which lack human interactions, there is a critical need to timely and automatically determine the localization of fire source [4]. Therefore, fire source localization technology has become an important topic in the fire detection field, and it also has significant research meaning for the identification of fire causes.

Nowadays, fire localization mainly uses image-based technology [5-8] and wireless sensor network-based technology [9-13]. Due to the susceptibility to shadowing, image-based technology is usually used in large open space. The wireless sensor network-based technology is

\footnotetext{
* Corresponding author: 1yczy299@126.com
}

based on the information of the fire characteristic physical quantities, such as the smoke concentration and the ambient temperature in the early stage of fire [14]. This method uses the diffusion model of the hot smoke flow in confined space, which can transform the fire source localization problem from three-dimensional space to twodimensional plane. Wang put forward the assumptions to treat the temperature flow as circular wavefronts, and proposed signal processing algorithms to resolve the problem of robust fire source localization [15]. Inspired by the capability of deep learning in data mining, Kou proposed a model based on gated recurrent unit to determine fire source localization and intensity [16]. Michaelides suggested the use of a sensor network for estimating the location of a source that releases certain substance in the environment, and then used nonlinear least squares optimization to estimate the source position based on the concentration readings at the sensor nodes [17]. Li proposed a fire intelligent monitoring and alarm system based on fuzzy neural network, which can provide more decision-making information for fixed-point firefighting [18].

However, all these reports did not change the point-bypoint output, which made it difficult to provide effective guidance for fire protection. Based on the diffusion law of hot smoke flow in confined space in the early stage of fire, 


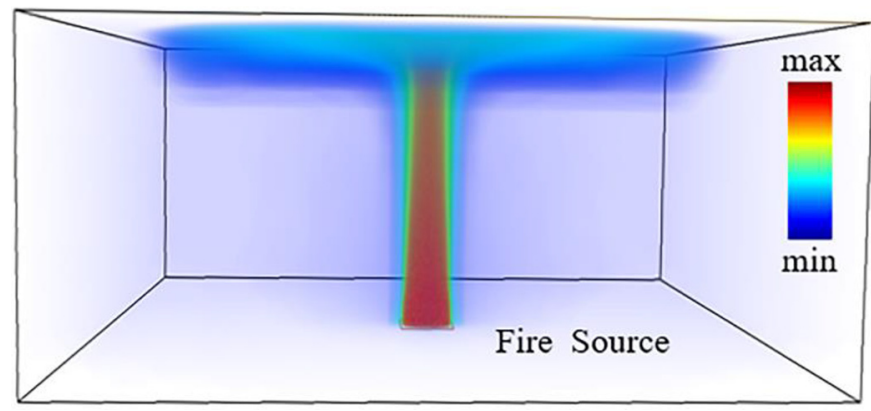

Fig. 1. Flow trend diagram of hot smoke flow in confined space.

this paper proposes a fire source localization method using the information of the fire characteristic physical quantities. This method makes full use of various fire information, and dynamically outputs the comprehensive localization results. The testing and analysis prove that the fire source localization method proposed in this paper can effectively select the localization result with an acceptable localization error.

\section{Diffusion law of hot smoke flow}

To use the sensors to localize the fire source in confined space, it is first necessary to clarify the development process of hot smoke flow. Normally, due to the low fire intensity source in the early stage, fire source can be considered as an ignition source that compared with the building scale. According to the knowledge of fire dynamics [19], the gas released by combustion is hotter than the surrounding air, thus forming a rising smoke plume. The heat flows along with the smoke plume, and a horizontally moving layer of hot smoke flow will be formed after the smoke plume reaches the ceiling.

Under the conditions without wind, the Gaussian model can be used to establish the diffusion model of hot smoke flow in confined space $[20,21]$, as shown in equation:

$$
S=S_{0}+C \cdot e^{-r^{2} / b}
$$

where, $r$ represents the distance between the fire source and a certain point on the ceiling, $S$ is the varying concentration of fire characteristic physical quantities at this point during the diffusion process, $S_{0}$ is the initial concentration of fire characteristic physical quantities in confined space, $C$ is the intensity of fire source, $b$ is the Gaussian distribution parameter.

It can be seen from equation (1) that when the hot smoke flow spreads on the ceiling, the concentration gradients of fire characteristic physical quantities are distributed in the annular waves. Figure 1 shows the flow trend of hot smoke flow in confined space, and the legend shows the intensity of hot smoke flow in different colors. Due to the surrounding cold air, hot smoke flow rises from the ignition point to the headspace, and then spreads around the ceiling. Therefore, the difference of fire characteristic physical quantity information at circular wavefronts can be used to localize the position of fire source.

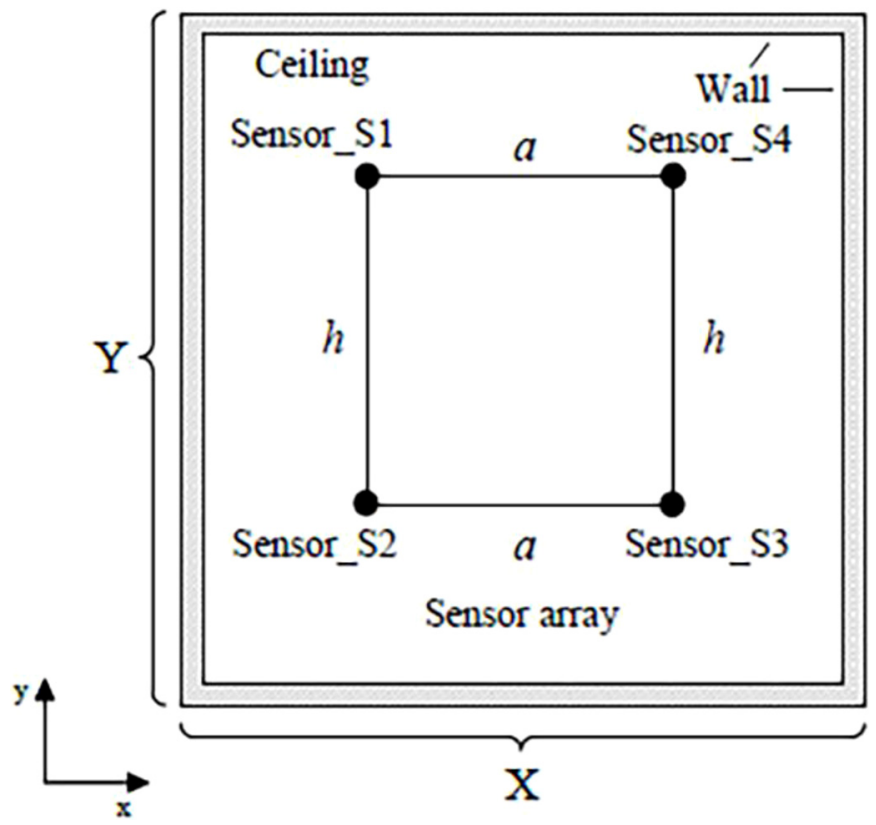

Fig. 2. Layout diagram of sensor array.

In order to emphasize the research priority, the research object and analysis model can be advisably simplified. According to the characteristics of the hot smoke flow and the previous researches $[15,16]$, following assumptions are proposed for the boundary conditions of fire source localization:

- The ceiling should be flat with a low thermal conductivity, and the initial temperature is basically the same.

- The current velocity of hot smoke flow should be approximately constant under the ceiling in the early stage of fire.

- The walls are of a similar temperature.

- The process of hot smoke flow rising and diffusing does not be restricted by external conditions.

Based on the above assumptions, the fire localization problem is transformed from a three-dimensional space to two-dimensional plane through the circular wavefronts on the ceiling of confined space.

\section{Fire source localization method}

\subsection{Basic principles of localization}

In view of the complex composition of hot smoke flow in the early stage of fire, we can select a sensor unit composed of smoke and temperature analog sensors. In actual monitoring, two types of sensors are used to localize the fire source at the same time, which can accurately reflect various fire situations in real time and provide more reliable information to improve the localization accuracy.

As shown in Figure 2, the length and width of the confined space are $\mathrm{X}$ and $\mathrm{Y}$ respectively. In the design of sensor array, a sensor array is composed of four sensor units. The geometric shape of array is a rectangle 
with length $a$ and width $h$, and four sensor units are S1, $\mathrm{S} 2, \mathrm{~S} 3, \mathrm{~S} 4$ at the vertex of the rectangle. Assuming the distance between the fire source and a sensor unit is $r$. Therefore, at each circular signal wavefront, the signal expectation that the sensor unit receives can be expressed as:

$$
f(t)=E\{S(r, t)\}_{r=\left(x-x_{0}\right)^{2}+\left(y-y_{0}\right)^{2}}, r \in R^{+}
$$

where $(x, y)$ is the position of any point on the ceiling, $\left(x_{0}, y_{0}\right)$ is the position of fire source, $E\{S(r, t)\}$ is the signal expectation of distance $r$ from the fire source.

Since hot smoke flow spreads around in the annular waves, the signal expectation of each point at the same circular wavefront is equal in the meantime, and function $S$ $(r, t)$ is only related to time $t$ and distance $r$. In each fixed fire condition, the position of sensor array is always known and the position of fire source will not change. Therefore, the distance $r$ between the fire source and the sensor unit is a fixed physical quantity.

Now it can be seen that the signal received by sensor unit is only a function of time $t$. The problem of localization can be reduced to a conventional time delay determination.

\subsection{Time delay based on the relative variability correlation degree}

Time delay is one of the important parameters that express signal characteristics [22]. Determining the time difference of the signals received by sensor units in the array is a prerequisite for calculating the distance and direction angle from the fire source. The basic idea of relative variability correlation degree is to judge whether the correlation of sequences is strong according to the similarity of sequence curve shapes. The closer the curve is, the stronger the correlation between the corresponding sequences is. Therefore, the relative variability correlation degree can be used to process the discrete signal sequences to obtain the correlations degree between different sequences, and then determine the time delay between the sensor units according to the order of correlation degree.

Combining with the actual conditions of scene, the thresholds of smoke concentration and ambient temperature should be preset in the sensor units. If at least one of the characteristic physical quantities meets the threshold, the four sensor units in the array will start collecting signal at the same time and obtain four corresponding groups of discrete signal sequences $S_{p}(n)$, where $p=1,2,3,4$. The discrete signal sequences $S_{p}(n)$ from the same fire characteristic physical quantity information can be expressed as:

$$
\left\{\begin{array}{l}
S_{1}(n)=\left\{S_{1}(1), S_{1}(2), \cdots \cdots\right\} \\
S_{2}(n)=\left\{S_{2}(1), S_{2}(2), \cdots \cdots\right\} \\
S_{3}(n)=\left\{S_{3}(1), S_{3}(2), \cdots \cdots\right\} \\
S_{4}(n)=\left\{S_{4}(1), S_{4}(2), \cdots \cdots\right\}
\end{array} .\right.
$$

Furtherly, we can intercept the sequence $S_{p}(k)$ of length $m$ from $S_{p}(n)$, and the length $m$ of sequence $S_{p}(k)$ can be determined by the sampling frequency of sensor units in actual monitoring. The interception of sequen-ces can dynamically correct the signal delay based on the obtained signal. Then, we can take $S_{1}(k)$ as the reference sequence, and take the remaining three groups of sequences $S_{i}(k)$ as the comparison sequence, where $i=2,3,4 . S_{i}^{\jmath}(k)$ is the time delay sequence with the comparison sequences $S_{i}(k)$ delayed by sample point number $j$, which can be expressed as:

$$
S_{i}^{j}(k)=S_{i}(k-j)
$$

where, the sample point number $j$ belongs to the set of integers, and the range of $j$ is $-t_{m} \leq j \leq t_{m}, t_{\mathrm{m}}$ is the maximum signal time delay. The reference sequence $S_{1}(k)$ and the time delay sequence $S_{i}^{j}(k)$ using the $l$ th segment of information are shown in following equations:

$$
\left\{\begin{array}{l}
S_{1}(k)=\left\{S_{1}((l-1) m+1), S_{1}((l-1) m+2), \cdots, S_{1}(l m)\right\} \\
S_{2}^{j}(k)=\left\{S_{2}^{j}((l-1) m+1), S_{2}^{j}((l-1) m+2), \cdots, S_{2}^{j}(l m)\right\} \\
S_{3}^{j}(k)=\left\{S_{3}^{j}((l-1) m+1), S_{3}^{j}((l-1) m+2), \cdots, S_{3}^{j}(l m)\right\} \\
S_{4}^{j}(k)=\left\{S_{4}^{j}((l-1) m+1), S_{4}^{j}((l-1) m+2), \cdots, S_{4}^{j}(l m)\right\}
\end{array} .\right.
$$

The correlation coefficient of relative variability between the reference sequence $S_{1}(k)$ and the time delay sequence $S_{i}^{j}(k)$ at point $k$ can be represented as:

$$
\zeta_{i}^{j}(k)=\frac{1}{1+\left|\frac{\Delta S_{1}(k)}{S_{1}(k)}-\frac{\Delta S_{i}^{j}(k)}{S_{i}^{j}(k)}\right|}
$$

where, $\frac{\Delta S_{1}(k)}{S_{1}(k)}, \frac{\Delta S_{i}^{j}(k)}{S^{j}(k)}$ represents the rate of relative change of the sequence at point $k, \triangle S_{1}(k)=S_{1}(k+1)-S_{1}(k)$ is the numerical difference between the reference sequence $S_{1}(k)$ at point $k+1$ and the point $k, \Delta S_{i}^{j}(k)=S_{i}^{j}(k+1)-S_{i}^{\jmath}(k)$ is the numerical difference between the time delay sequence $S_{i}^{j}(k)$ at point $k+1$ and point $k$.

The relative variability correlation degree between the reference sequence and the time delay sequence can be expressed as:

$$
r_{i}^{j}=\frac{1}{m-1} \sum_{k=1}^{m-1} \zeta_{i}^{j}(k)
$$

Furtherly, we can sort the relative variability correlation degree between the reference sequence and different time delay sequence, and then select the time delay sequence with the largest $r_{i}^{j}$, the corresponding $j$ is the delayed sample point number between the reference sequence $S_{1}(k)$ and the comparison sequence $S_{i}(k)$. From this, we can get:

$$
t_{1 i}=\frac{j}{f_{A}}
$$

where, $t_{1 i}$ is the real time delay between the two corresponding sensor units, $f_{A}$ is the sampling frequency of sensor units.

Similarly, we can intercept different segments of the sequence $S_{p}(k)$ of length $\mathrm{m}$ in the discrete signal sequence $S_{p}(n)$, and then new signal time delay can be obtained by calculating. 


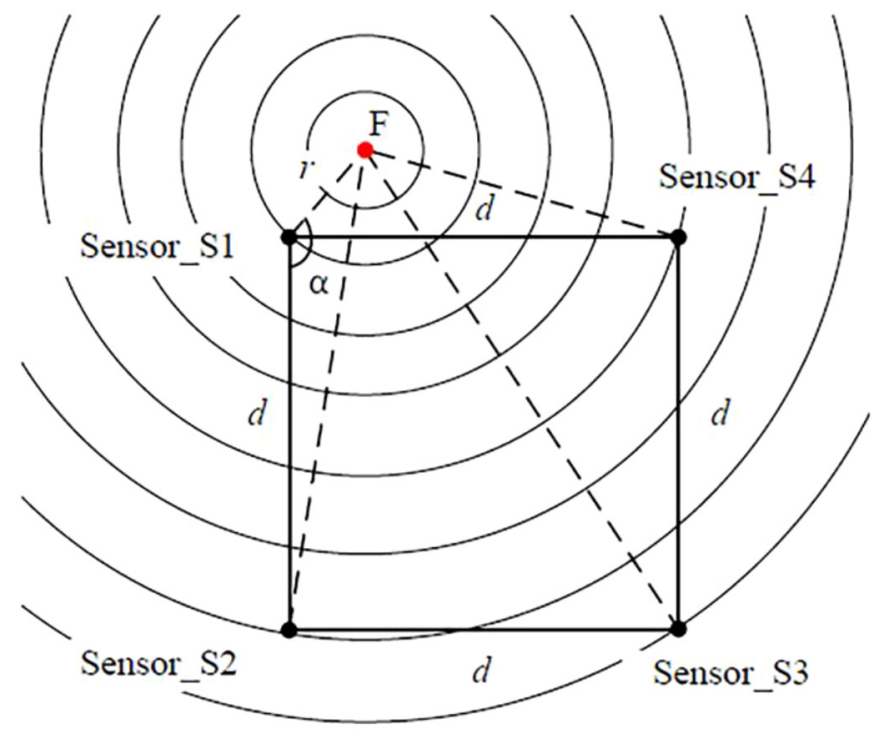

Fig. 3. Schematic diagram of sensor array localization.

\subsection{Angular localization}

In view of the layout of sensor array described above, the specific principle of the angular localization of fire source will be explained in this section. Assuming the geometric shape of sensor array is square with the side length $d$, and the sensor unit $\mathrm{S} 1$ is taken as the reference sensor unit. The four sensor units are arranged in an anticlockwise direction in the array, as shown in Figure 3.

According to the geometric relationship between the fire source and the sensor array, from the law of cosines, the equations can be got as follow.

$$
\left\{\begin{array}{l}
\left(v t_{12}+r\right)^{2}=r^{2}+d^{2}-2 r d \cos \alpha \\
\left(v t_{14}+r\right)^{2}=r^{2}+d^{2}-2 r d \cos \left(\frac{\pi}{2}-\alpha\right) \\
\left(v t_{13}+r\right)^{2}=r^{2}+2 d^{2}-2 \sqrt{2} r d \cos \left(\alpha-\frac{\pi}{4}\right)
\end{array}\right.
$$

where, $v$ is the diffusion velocity of hot smoke flow, $r$ is the distance between the reference sensor unit S1 and the fire source $F, \alpha$ is the direction angle that created by the square side S1 S2 and side S1 F, $t_{12}, t_{13}$, and $t_{14}$ are the signal time delays that the signal wavefronts passing through the sensor units S1 and S2, S1 and S3, S1 and S4, respectively.

It can be seen that equations (9) is a nonlinear equation set about the diffusion velocity $v$, the direction angle $\alpha$ and the distance $r$. After solving this equation set, the direction angle $\alpha$ between the fire source and the sensor unit S1 can be obtained. In the same way, by selecting different sensor units as the reference sensor units, we can respectively calculate the direction angles between the fire source and the sensor units S2, S3, S4. Then, two direction angles will be used to perform angular localization, the result is shown in Figure 4.

Furtherly, to enhance the robustness of the method and reduce the localization error, we can use the data information provided by sensor units as much as possible. Under one set of time delays, the fire source localization

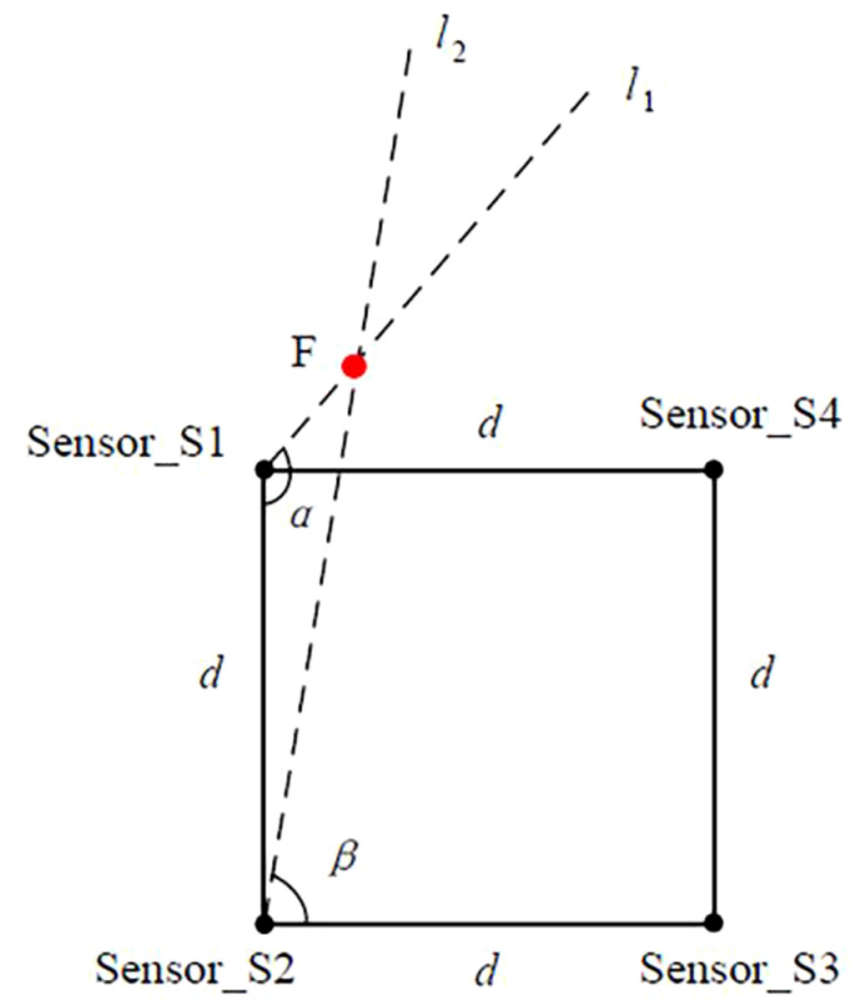

Fig. 4. Angular localization by sensor unit.

results determined by the angular localization of any two sensor units in the array are recorded in the set $\mathrm{M}$, and the average value of all results in the set $M$ is taken to obtain the optimal localization result $x_{i}$.

\subsection{Comprehensive localization of fire source based on dynamic clustering}

With the development of fire, new time delays can be calculated by the increasing discrete signal sequences. Then, new fire source localization results will also continue to be obtained. In order to improve the localization accuracy, we can use the dynamic clustering to further analyze and refine the results to obtain a more accurate comprehensive localization result.

The traditional clustering method requires all sample data to be clustered, which has the dis-advantage of not being able to iterate. However, in this paper, the fire source localization results are point-by-point input over time, thus the traditional method is difficult to apply. To aim at the characteristics of point-by-point input, we can perform dynamic clustering iterative calculation on the fire source localization results input, then use the result of clustering to select a localization estimation area, and further consider the center of the localization estimation area as the dynamic output of the comprehensive localization result. In addition, since the sum of squares of errors is constantly changing during the iteration process, the variation of the sum of squares of errors can be used as the termination condition of the algorithm. The specific process is shown in Figure 5: 


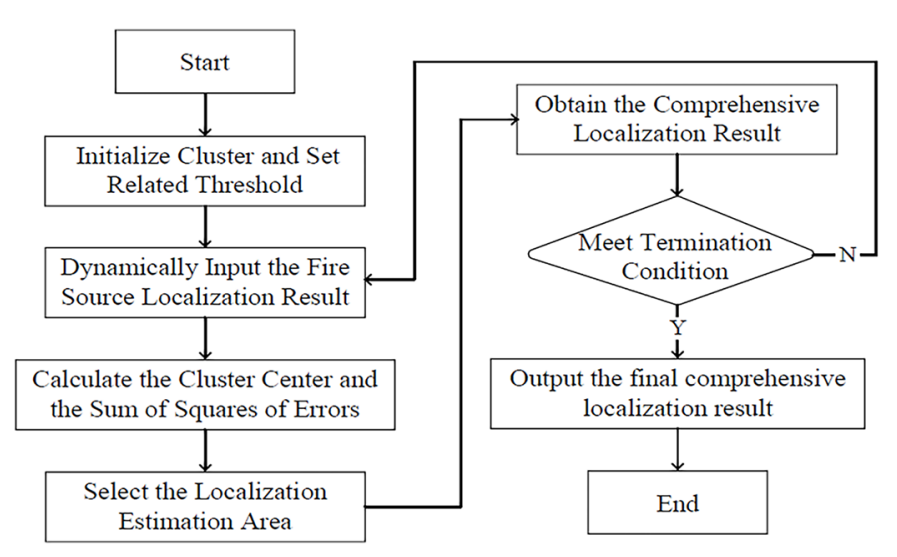

Fig. 5. Flow chart of comprehensive localization of fire source.

- Initialize the number of input samples $N=0$, and consider the first input of the fire source localization results as the initial cluster center $Z$.

- Dynamically input the fire source localization result $x_{\mathrm{i}}$, set the number of input samples to $N+1$, and recalculate the cluster center $Z$, as shown in the equation:

$$
Z=\frac{1}{N+1} \sum_{i=1}^{n} x
$$

- Calculate the sum of squares of errors $I_{\mathrm{E}}$ between the cluster center $Z$ and the input fire source localization results at this time, as shown in the equation:

$$
I_{E}=\sum_{i=1}^{n}\left(x_{i}-Z\right)^{2}
$$

- Set the distance threshold $W_{1}$, and take the area with the distance $W_{1}$ from the cluster center $Z$ as the localization estimation area.

- Take the average value of all the results in the estimation area to obtain the comprehensive localization result, and output the result dynamically.

- Set the error threshold $W_{2}$, if the absolute value of the difference between the current and the previous sum of squares of errors is less than the threshold $W_{2}$, stop the iteration and determine the final comprehensive localization result of the fire source.

\section{Testing and analysis}

In order to verify the feasibility of the proposed localization method and determine the error of the method, this paper uses the fire dynamics numerical simulation software PyroSim to build a confined space with a building size of $20 \mathrm{~m} \times 20 \mathrm{~m} \times 4 \mathrm{~m}$. As the simulation model shown in Figure 6 , the coordinate of the fire source is set to $(6,8)$, the simulation type is selected as the common unsteady fire model. The maximum heat release rate $Q_{\max }$ is set to $6 \mathrm{MW} / \mathrm{m}^{2}$, and the initial ambient temperature is set to $20^{\circ} \mathrm{C}$.

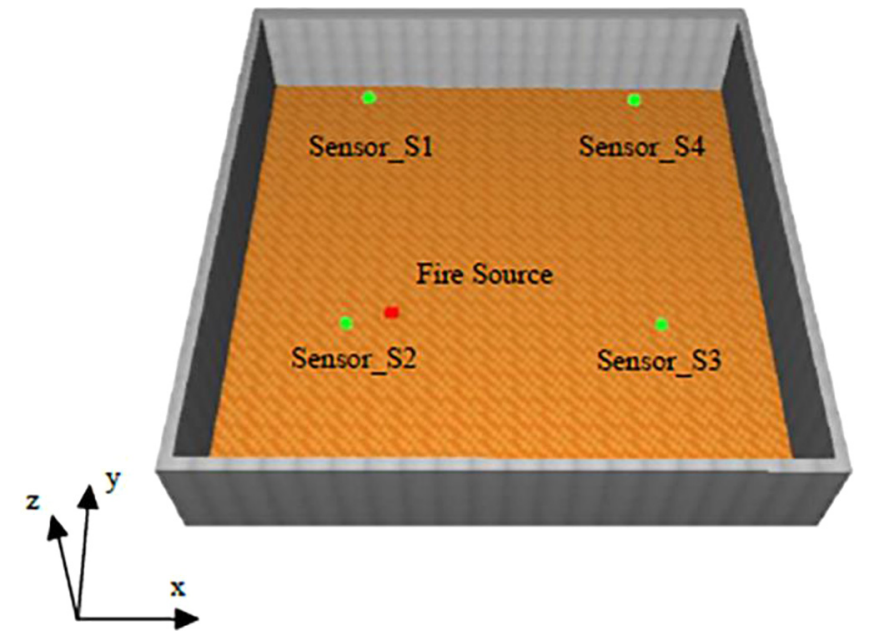

Fig. 6. The diagram of simulation model.

The sensor array is arranged on the ceiling in the confined space, with the size of $10 \mathrm{~m} \times 10 \mathrm{~m}$, and the coordinates of the four sensor units in the array are $(5,5)$, $(5,15),(15,5)$ and $(15,15)$, respectively. Each sensor unit is equipped with two types of sensors, smoke and temperature, with the simulation signal sampling frequency of $50 \mathrm{~Hz}$. According to the technical regulations for building fire prevention, the smoke sensor threshold is set to $100 \mathrm{ppm}$, and the temperature sensor threshold is set to $50^{\circ} \mathrm{C}$. The smoke signal and temperature signal collected by the sensor units are shown in Figure 7.

According to the generated simulation signal, the time when the threshold is reached is taken as the starting time point. Considering the simulation signal sampling frequency, the discrete signal sequences with the length of $m=1000$ are intercepted. Then, the relative variability correlation degree method is used to obtain the signal time delays between the sensor units. As time passing by, new discrete signal sequence is re-intercepted per $2 \mathrm{~s}$ after the starting time point, thus new signal delays are continuously calculated, and then the fire source localization results under different sets of delay conditions that vary with time can be obtained.

Considering the aspect of localization accuracy, the distance threshold $W_{1}$ is set to $1.2 \mathrm{~m}$. Based on the idea of dynamic clustering, a localization estimation area changing with the input of fire source localization results is generated. The average value of all the results in the estimation area is used as the result of the comprehensive localization result of the fire source. Figure 8 shows the result obtained when the number of input samples is 50 . From this we can see, the coordinate of the cluster center is $(6.32,7.74)$, and the coordinate of the comprehensive localization result are $(6.15,7.86)$. It can be seen that the localization results dynamically input over time basically appear near the actual fire source. In addition, the localization estimation area can also well exclude the scattered samples, and select the area with the densest samples. 


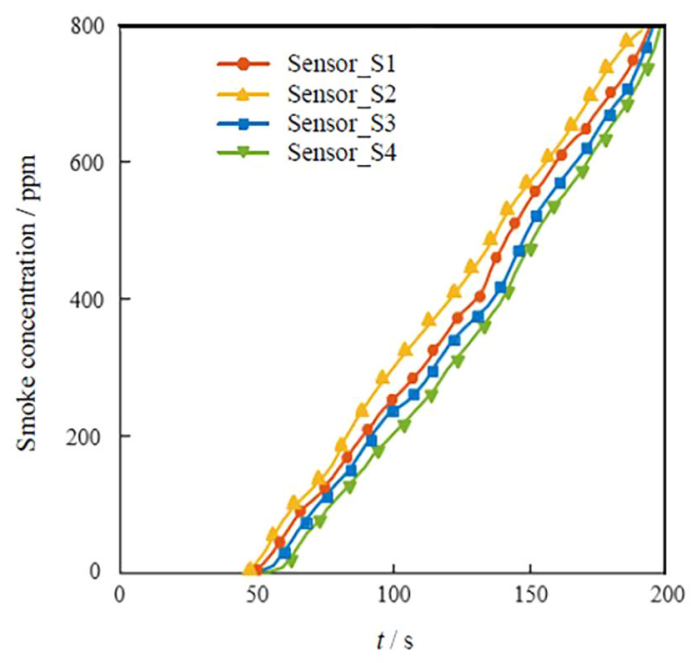

(a)

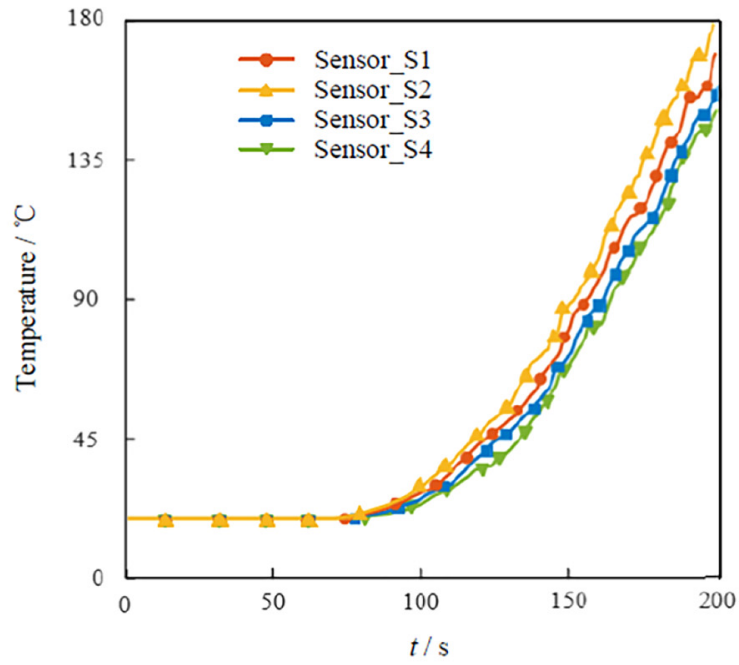

(b)

Fig. 7. Signal collected by the sensor units. (a) Smoke signal. (b) Temperature signal.

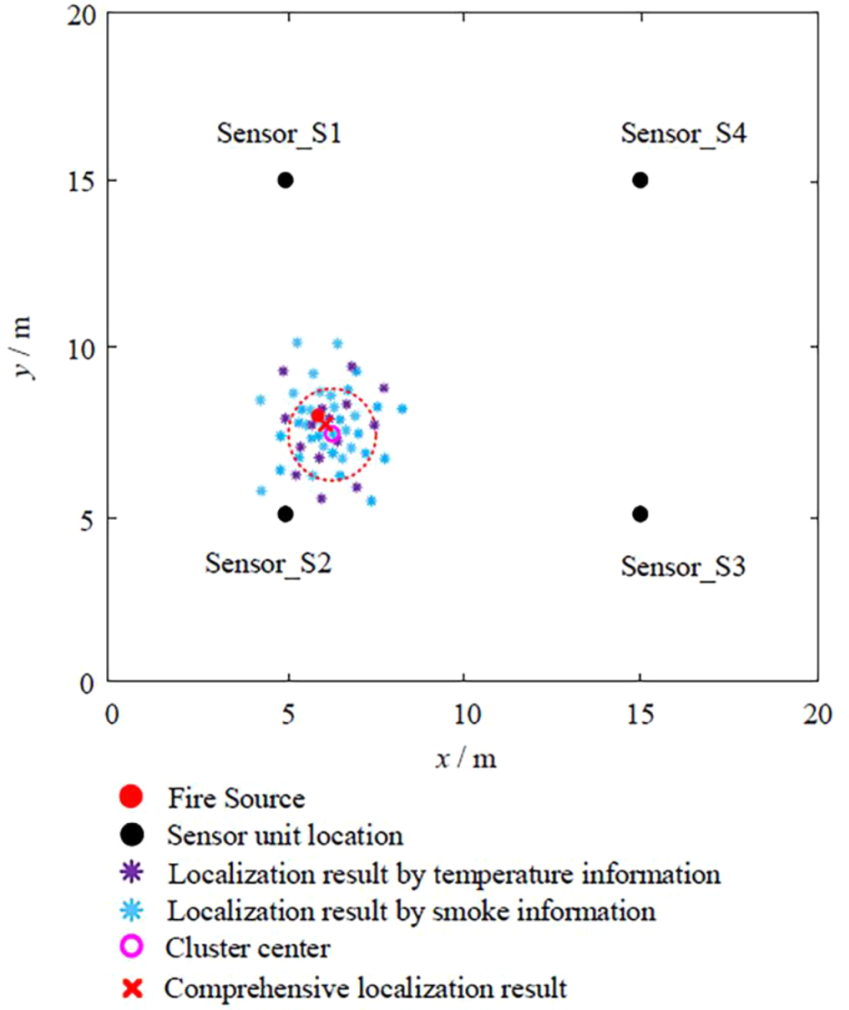

Fig. 8. Comprehensive localization results of fire source.

The comprehensive localization result obtained according to the estimation area is also near the actual position of the fire source, and the distance between both of them can be expressed by the localization error, which can be got as follow:

$$
\sigma=\sqrt{\left(x(i)-x_{0}\right)^{2}+\left(y(i)-y_{0}\right)^{2}} .
$$

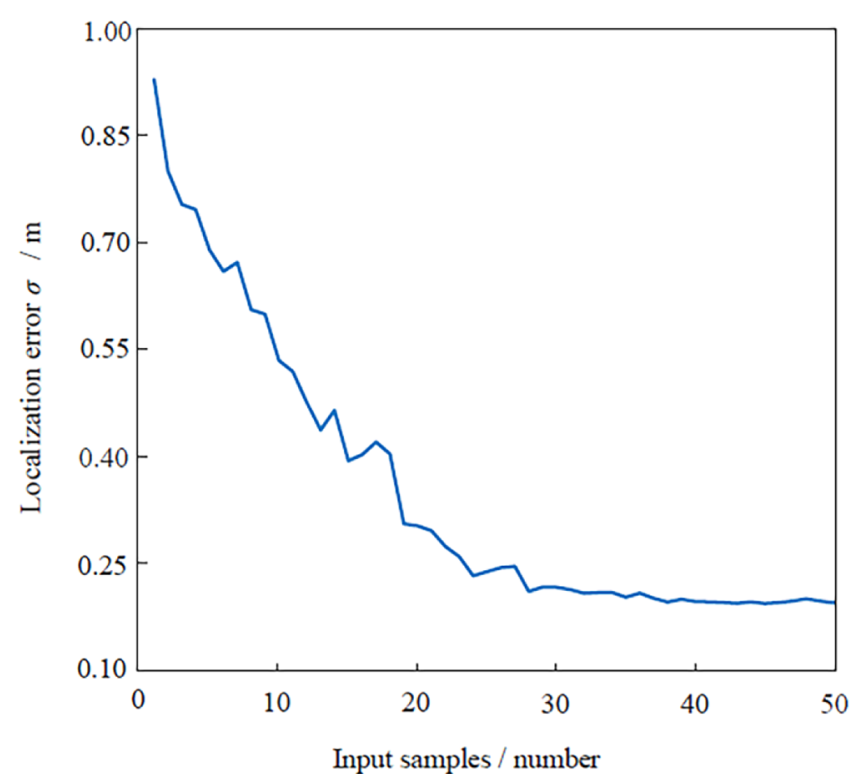

Fig. 9. Trend diagram of localization error.

As the number of input samples increases, the trend of localization error is shown in Figure 9.

It can be seen that the localization error basically decreases as the number of input samples increases, and finally stabilizes at about $0.21 \mathrm{~m}$.

Changing the position coordinate of the fire source, the comprehensive localization results and errors are shown in Table 1. From the analysis of the testing results, it can be found that each tests can effectively determine the position of the fire source, and the range of localization error is within $0.3 \mathrm{~m}$.

The testing results demonstrate the feasibility and stability of the method in this paper. It is an excellent algorithm suitable for the localization of fire source in the early stage of fire, which can effectively improve the localization accuracy. 
Table 1. Comprehensive localization results and localization errors of the different fire sources, Unit: $\mathrm{m}$.

\begin{tabular}{lll}
\hline Actual fire source & Comprehensive localization result & Localization error \\
\hline$(6.00,3.00)$ & $(5.83,2.92)$ & 0.19 \\
$(7.00,5.50)$ & $(7.19,5.34)$ & 0.25 \\
$(8.00,6.50)$ & $(7.90,6.24)$ & 0.28 \\
$(9.00,9.00)$ & $(9.08,9.12)$ & 0.14 \\
$(10.50,9.00)$ & $(10.43,8.94)$ & 0.09 \\
$(12.00,15.00)$ & $(12.19,14.87)$ & 0.22 \\
$(14.00,10.00)$ & $(13.84,10.08)$ & 0.18 \\
$(16.50,7.00)$ & $(16.60,6.79)$ & 0.23 \\
\hline
\end{tabular}

\section{Conclusion}

This paper first describes the diffusion law of hot smoke flow in the early stage of fire, and then proposes a fire source localization method in confined space using the information of the fire characteristic physical quantity. For the purpose of reflect various fire situations accurately in real time, the sensor unit is composed of the smoke and temperature sensors. According to the relative variability correlation degree, time delay between the sensors can be obtained by the collected fire characteristic physical quantity signals, After that, the calculated direction angles are used to perform angular localization to obtain fire source localization results. Finally, based on the idea of dynamic clustering, the average value of all the results in the estimation area is selected as the comprehensive localization result. In general, this method can continuously correct the time delay signals between the sensors, avoiding the excessive dependence of localization accuracy on the consistency of the time delay signal. It also overcomes the initial need for all samples in the traditional clustering, and can be performed iteratively.

The analysis of the testing results shows that the proposed method can effectively select the localization estimation area containing the fire source, and the localization error between the comprehensive localization result and the actual fire source is within the acceptable. The accuracy and robustness can be guaranteed to provide better guidance for designated fire prevention and cause identification. Relevant research will continue to be carried out in the next step, the method will be further improved to enhance the localization accuracy, and to adapt to more complex fire scenes.

Acknowledgment. This work is supported by National Science Foundation of China (Grant Nos. 52077221), and Science and Technology Project of State Grid Hubei Electric Power Co., Ltd (No. 52153220001V).

\section{References}

1. V. Hristidis, S.C. Chen, T. Li, S. Luis, Y. Deng, Survey of data management and analysis in disaster situations, J. Syst. Softw. 83, 1701-1714 (2010)
2. Z. Bi, T. Le, H. Pan, H. Yang, Y. Jiang, Overview on the preprocessing method of image fire detection, Fire. Sci. Technol. 35, 87-91 (2016)

3. N.D. Hansen, F. Steffensen, M. Valkvist, G. Jomaas, R. Van Coile, A fire risk assessment model for residential high-rises with a single stairwell, Fire. Saf. J. 95, 160-169 (2018)

4. R. Bogue, Sensors for fire detection, Sensor. Rev. 33, 99-103 (2013)

5. S. Duan, D. Mao, Research on fire flame image detection algorithm, Comput. Simulat. 33, 393-398 (2016)

6. M. Lin, W. Chen, B. Liu, An intelligent fire-detection method based on image processing, Adv. Eng. Forum. 2, 172-175 (2011)

7. A.K. Muhammad, J. Ahmad, W. Sung, Early fire detection using convolutional neural networks during surveillance for effective disaster management, Neurocomputing 288, 30-42 (2018)

8. P. Foggia, A. Saggese, M. Vento, Real-time fire detection for video-surveillance applications using a combination of experts based on color, shape, and motion, IEEE Trans. Circuits Syst. Video Technol. 25, 1545-1556 (2015)

9. Y. Mao, H. Wang, Y. Lu, L. Qin, Precise localization method for fire sources in large-space buildings, Comput. Appl. Softw. 33, 169-172 (2016)

10. D. Li, Q. Liu, Y. He, H. Wang, L. Hu, Research on data fusion algorithm of fire detection signal in confined space, Fire. Sci. Technol. 40, 164-168 (2021)

11. Y. He, Y. Dong, J. Lei, W. Zhang, R. Xue, Research on multisensor method for detecting aircraft cargo fire, Chin. Saf. Sci. J. 28, 74-79 (2018)

12. Y. Kong, C. Lang, S. Feng, T. Wang, M. Yin, Fire detection based on two-stream ordinal regression deep network consumed, Chin. Sci. Pap. 12, 1590-1595 (2017)

13. J. Fonollosa, A. Solórzano, S. Marco, Chemical sensor systems and associated algorithms for fire detection: a review, Sensors-Basel. 18, 553-553 (2018)

14. A. Gaur, A. Singh, A. Kumar, Fire Sensing Technologies: A Review, IEEE Sensors. J. 19, 3191-3202 (2019)

15. S. Wang, M. Berentsen, T. Kaiser, Signal processing algorithms for fire localization using temperature sensor arrays, Fire. Saf. J. 40, 689-697 (2005)

16. L. Kou, X. Wang, X. Guo, J. Zhu, H. Zhang, Deep learning based inverse model for building fire source location and intensity estimation, Fire. Saf. J. 121, 1-10 (2021)

17. M. Michaelides, C. Panayiotou, Plume source position estimation using sensor networks, Proceedings of the 13th Mediterranean Conference on Control and Automation Limassol, IEEE, 2005, pp. 731-736 
18. Z. Li, P. Miao, Y. Liu, Fire detection and position algo-rithm for large room based on wireless sensor network, J. Data. Acquis. Process. 29, 964-969 (2014)

19. G.S. Birajdar, M. Baz, R. Singh, M. Rashid, A. Gehlot, S.V. Akram, S.S. Alshamrani, A.S. AlGhamdi, Realization of people density and smoke flow in buildings during fire accidents using raspberry and openCV, Sustainability-Basel 13, 11082-11082 (2021)
20. L. Wu, H. Lei, D. Huang, G. Chen, X. Lu, Y. Yang, Smoke detection algorithm based on Gaussian mixture motion detection model and multiple features, Autom. Inf. Eng. 35, 1-5 (2014)

21. Z. Liu, Diffusion model for fire smoke based on Gaussian distribution, Fire. Sci. Technol. 33, 1205-1207 (2014)

22. G. Susto, A. Cenedese, M. Terzi, Time-series classification methods: Review and applications to power systems data, Big. Data. Appl. Pow. Syst. 9, 179-220 (2018)

Cite this article as: Fan Yang, Zhuoyuan Cai, Lei Su, Yongduan Xue, Yu Shen, Junjie Wang, Research on fire source localization in confined space based on the fire characteristic physical quantity information, Int. J. Metrol. Qual. Eng. 13, 1 (2022) 